\title{
Effects of Geometrical-Size of Cylindrical-Shell Transducer on Acoustic- Beam Steering Efficiency for a Slim-Hole Acoustic-Logging Tool
}

\author{
Lin $\mathrm{Fa}^{*}, 1$, Zhengwen $\mathrm{Zeng}^{2}$, Chuanbao Deng ${ }^{3}$ and Meishan Zhao ${ }^{4}$
}

\author{
${ }^{I}$ Department of Electronics and Information Engineering, Xi'an Institute of Post and Telecommunications, Weiguo \\ Road, Xi'an, Shaanxi 710121, P.R. China \\ ${ }^{2}$ Department of Geology and Geological Engineering, The University of North Dakota, P.O. Box 8358, Grand Forks, \\ ND 58202-8358, USA \\ ${ }^{3}$ Department of Electric and Computer Engineering, The University of Wisconsin-Madison, 1415 Engineering Drive, \\ Madison, WI 53706, USA \\ ${ }^{4}$ The James Franck Institute and Department of Chemistry, The University of Chicago, 5735 South Ellis Avenue, \\ Chicago, IL 60637, USA
}

\begin{abstract}
The slim-hole acoustic-logging tool is often used for measurement while drilling and horizontal well logging. The source and receiver are generally thin cylindrical-shell piezoelectric transducers. The radius of the drilling-collar limits the size of the cylindrical-shell transducer in the logging tool. The smaller the radiation area of the transducer, the smaller the radiated acoustic energy, and the smaller its radius, the higher the frequency of the radiated acoustic signals. Besides, the attenuation for higher frequency wave propagating in the medium is higher. Due to these reasons the amplitude of the measured acoustic signal by using the slim-hole logging tool is usually much smaller than that by using the conventional logging tool. Therefore, the acoustic-beam steering technology is important for enlarging the amplitude of the received acoustic signal during logging with a slim-hole logging tool. The geometrical-size of the cylindrical-shell transducer influences the acoustic-beam steering efficiency of the logging tool with a line-array source and a receiver. In this paper, in the frame work of the acoustic-logging transmission network model with the concept of directivity-weighted coefficient, we have carried out the calculation and analysis of the effects of geometrical-size of the transducer on the acoustic-beam steering efficiency of the slim-hole acoustic-logging tool with a line-array source and a receiver. The calculated results are useful for optimizing the design of the slim-hole acoustic-logging tool with a line-array source and a receiver.
\end{abstract}

Keywords: Slim-hole, cylindrical-shell transducer, acoustic-logging, electric-acoustic transmission network, acoustic-electrical transmission network.

\section{INTRODUCTION}

Almost all the practical sources and receivers in acousticlogging tools are thin cylindrical-shell piezoelectric transducers polarized in their radius direction. Unlike the omnidirectional acoustic radiation of the thin spherical-shell transducer polarized in its radius direction, the acoustic radiation and acceptance of the cylindrical-shell transducer have their preferred directivities, which depend on the geometrical-size of the transducer. In most previous acoustic-logging modeling, ideal signals or other mathematical expressions such as Ricker wavelet [1], Tsang wavelet [2, 3], Gaussian impulse wavelet [4], as well as other various wavelets are usually used to describe the acoustic source. Furthermore, they are considered to be the omnidirectional point source. Fa et al. [5-7] used a thin spherical-shell transducer polarized in radius direction as the

*Address correspondence to this author at the Department of Electronics and Information Engineering, Xi'an Institute of Post and Telecommunications, Weiguo Road, Xi'an, Shaanxi 710121, P.R. China; Tel: 0086-29-88166264;

E-mail: fa_yy@yahoo.com.cn equivalent to an omnidirectional point source in acousticlogging. These researches have neglected the effects of directivity of the practical cylindrical-shell transducer (which is used as the source or the receiver) on the measured acoustic-logging signal.

During measurement while drilling or horizontal well logging, the radius of the drilling-collar limits the size of the cylindrical-shell transducer in the logging tool. Attempts to utilize the conventional logging pattern with a source and a receiver for slim-hole acoustic-logging are often unsuccessful due to weak signal amplitudes. Reasons for poor signal include: (1) smaller transducer radius generates higher frequency signals which attenuate more rapidly; (2) the radiation area of the transducer decreases with its diameter, so the radiated acoustic energy decreases outwards; (3) because the logging tool is installed in a steel sleeve with slots, scattering reduces the received acoustic signals; and (4) the radiated acoustic energy in the direction of incident critical-angle from the drilling mud to the formation decreases with the ratio of radius to height for the cylindrical-shell transducer due to greater concentration of 
the radiated acoustic energy normal to the borehole wall. To increase the effective energy radiated by the source and the amplitude of received acoustic signal, the acoustic-beam steering technology should be applied in the slim-hole acoustic-logging tools. On the basis of acoustic-logging transmission network model [6], we use the concept of the directivity-weighted coefficient to calculate, analyze and discuss the effects of the geometrical-size of the transducer on the beam steering efficiency of the slim-hole acousticlogging tool with a line-array source and a single receiver. In this paper we present an analysis method of the acousticbeam steering efficiency for the slim-hole logging tool. The calculated results verify that properly choosing the geometrical-size of the cylindrical-shell transducer can improve the acoustic-beam steering efficiency and increase the amplitude of the received acoustic signal for the slimhole logging tool.

\section{MODELING}

In this section, we use the concept of directivity-weighted coefficient and the acoustic-logging transmission network model to calculate the effects of the transducer directivity on the acoustic-beam steering efficiency for the slim-hole logging tool.

\subsection{The Directivity of Cylindrical-Shell Transducer}

Let the thickness, height and average radius of the cylindrical-shell transducer polarized in radius direction be $l_{t}, H$ and $r_{b}$, respectively. Usually, transformer oil is used as the coupling medium around the transducer in the logging tool; its acoustic velocity $v_{m}$ and density $\rho_{m}$ are $1425 \mathrm{~m} / \mathrm{s}$ and $856.5 \mathrm{~kg} / \mathrm{m}^{3}$. Generally, both the transducers (source and receiver) and the transformer oil are isolated from the borehole fluid with a thin rubber pocket. Because the thickness of the rubber pocket is very small, the created acoustic reflection and attenuation are also very small when the acoustic signals pass through it. The rubber pocket can be approximately considered to be an acoustic penetrating layer. Because the acoustic velocity in the borehole fluid is about equal to that in the transformer oil, the directivity of the transducer in the transformer oil can be approximated by the directivity in the borehole fluid. The directivity of the cylindrical-shell transducer can be described as $[8,9]$

$$
G(\theta)=\left[\frac{J_{0}^{2}\left(\frac{2 \pi r_{b}}{\lambda} \cos \theta\right)+\cos ^{2} \theta J_{1}^{2}\left(\frac{2 \pi r_{b}}{\lambda} \cos \theta\right)}{J_{0}^{2}\left(\frac{2 \pi r_{b}}{\lambda}\right)+J_{1}^{2}\left(\frac{2 \pi r_{b}}{\lambda}\right)}\right]^{1 / 2} \frac{\sin \left(\frac{\pi H}{\lambda} \sin \theta\right)}{\frac{\pi H}{\lambda} \sin \theta},
$$

where, $J_{0}$ is the zeroth-order Bessel function, $J_{1}$ is the firstorder Bessel function, $\lambda$ is the wavelength of acoustic wave in the coupling fluid (or in the borehole fluid) and $\theta$ is the angle between the propagation direction of the acoustic signal radiated by the cylindrical transducer and the direction perpendicular to the wall of the transducer.

\subsection{Electrical-Acoustic and Acoustic-Electrical Transmission Networks of the Transducer}

Fig. (1a, b) are the electrical-acoustic and acousticelectrical transmission networks for the cylindrical-shell transducer, respectively $[5,10]$. Here, $R_{1}$ is the output resistance of the driving circuit; $U_{1}(t)$ is the driving-voltage signal; $V(t)$ and $X(t)$ are the electrical voltage input and acoustic pressure output signals of the source; $R_{3}$ is the input resistance of the measurement circuit; $P(t)$ and $U_{3}(t)$ are the acoustic pressure input and electrical output signals of the receiver; $N_{i}, C_{i 0}, C_{i m}, m_{i}, R_{i r}, m_{i r}$ and $R_{i m}$, are the mechanical-electrical conversion coefficient, 'restraint' static capacitance, elastic stiffness, mass, radiation force resistance, radiation mass and friction force resistance of the transducer, respectively; and $i=1$ stands for the source and $i=3$ stands for the receiver.

(a) Electrical-acoustic transmission network (source)

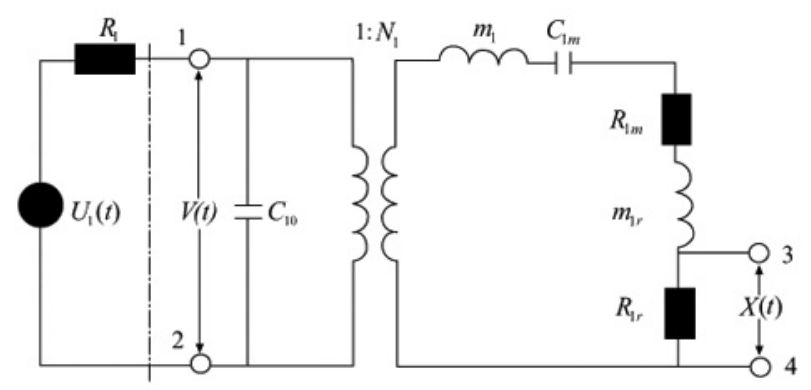

(b) Acoustic-electrical transmission network (receiver)

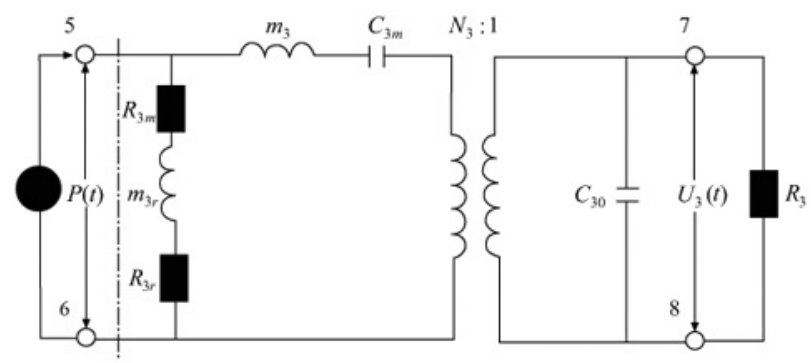

Fig. (1). Equivalent circuit of thin-shell cylindrical transducer. Where $\quad N_{i}=2 \pi H_{i} d_{i 31} / s_{i 11}^{E}, \quad C_{i 0}=2 \pi r_{i b} H_{i} \varepsilon_{i 33}^{T}\left(1-k_{i p}^{2}\right) / l_{i t}$, $C_{i m}=r_{i b} s_{i 11}^{E} /\left(2 \pi H_{i} l_{i t}\right), \quad m_{i}=2 \pi r_{i b} H_{i} l_{i t} \rho_{i}, \quad R_{i m}=0.4 \pi r_{i b} H_{i} \rho_{m} v_{m}$, $R_{i r}=2 \pi H_{i} k_{i}^{2} r_{i b}^{3} \rho_{m} v_{m} /\left(1+k_{i}^{2} r_{i b}^{2}\right), \quad m_{i r}=2 \pi H_{i} \rho_{m} v_{m} r_{i b}^{2} /\left(1+k_{i}^{2} r_{i b}^{2}\right)$, $k_{i p}=d_{i 31} / \sqrt{s_{i 11}^{E} \varepsilon_{i 11}^{T}}$, and $k_{i}=\omega_{i} / v_{m} ; \rho_{i}, s_{i 11}^{E}, d_{i 31}$ and $\varepsilon_{i 33}^{T}$ are the density, strain, piezoelectric and dielectric constants of the transducer material, respectively; $r_{i b}, H_{i}, r_{i b}$ and $l_{i t}$ are the average radius, height and wall thickness of the transducer, respectively.

When the cylindrical-shell transducer is used as a source as shown in Fig. (1a), the electrical-acoustic impulse response and the loading center frequency can be written as (see Appendix A)

$h_{1}(t)=K_{11} e^{-\alpha_{1} t}+2 K_{12} e^{-\beta_{1} t} \cos \left(\omega_{1} t+\theta_{1}\right)$,

and

$f_{1}=\omega_{1} / 2 \pi=\sqrt{3} B_{1} / 2 \pi=\sqrt{3}\left(x_{1}-y_{1}\right) / 4 \pi$.

When the transducer is used as a receiver as shown in Fig. (1b), the acoustic-electrical impulse response and the loading center frequency can be written as (see Appendix B) 
$h_{3}(t)=K_{31} e^{-\alpha_{3} t}+2 K_{32} e^{-\beta_{3} t} \cos \left(\omega_{3} t+\theta_{3}\right)$,

and

$f_{3}=\omega_{3} / 2 \pi=\sqrt{3} B_{3} / 2 \pi=\sqrt{3}\left(x_{3}-y_{3}\right) / 4 \pi$.

The electrical terminals of the source are the input terminals and its mechanical terminals are the output terminals, with the converse for the receiver. Therefore, even if the physical and geometrical parameters of both the source and the receiver are the same, the electrical-acoustic transmission property of the source is somewhat different from the acoustic-electrical transmission property of the receiver; and the loading center frequency, $f_{3}$, of the receiver is not equal to the loading center frequency, $f_{1}$, of the source.

\subsection{Filtering Effect of Propagation Media on Logging Signals}

Suppose the logging tool consists of a receiver $J$ and a line-array source. The line-array source is comprised of $n$ cylindrical-shell transducer elements $\left(T_{1}, T_{2}, \ldots \ldots T_{n}\right)$. For a fluid-filled cylindrical-borehole of radius $R$ embedded in an infinite elastic medium, the lowest transducer element $T_{1}$ in the line-array source is located at the origin as shown in Fig. (2).

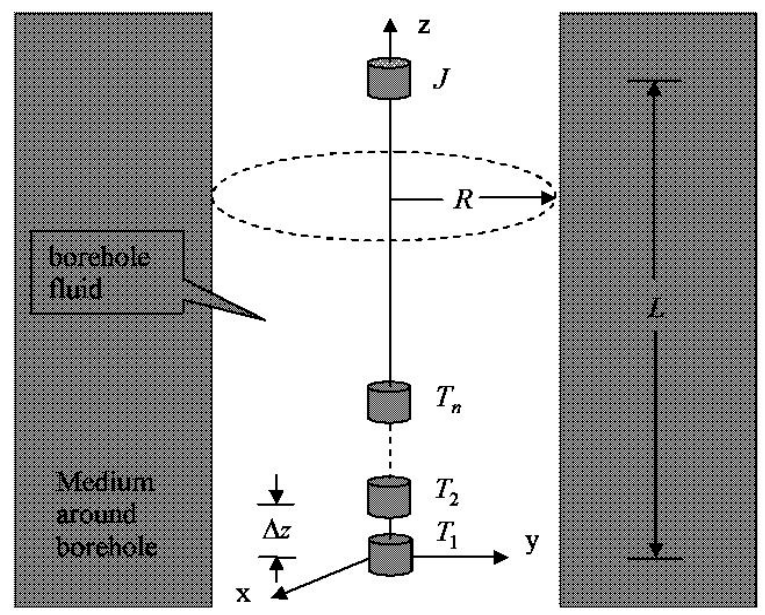

Fig. (2). Configuration of acoustic-logging for a slim-hole acousticlogging tool with a line-array source and a receiver. Where $\Delta z$ is the interval between two neighboring transducer elements in the line-array source, and $L$ is the separation from the source to the receiver.

The transmission function and impulse response of the media (the borehole fluid and formation around the borehole) for the acoustic signals radiated by each transducer element in the line-array source can be described by $[6,11]$

$$
\begin{aligned}
& H_{2 l}(\omega)=H_{2 d l}(\omega)+H_{2 r l}(\omega) \\
& =\frac{1}{z-l \Delta Z} e^{-j \omega\left\{l \Delta t-[z-(l-1) \Delta Z] V_{f}\right\}}+\frac{1}{(2 \pi)^{2}} e^{-j \omega(l-1) \Delta t} \int_{-\infty}^{+\infty} A\left(k_{z}, \omega\right) e^{j k_{z}[z-(l-1) \Delta z]} d k_{z},
\end{aligned}
$$

and

$$
h_{2 l}(t)=\int_{-\infty}^{+\infty} H_{2 l}(\omega) e^{j \omega t} d \omega,
$$

where, $k_{z}$ is axial wavenumber; $\Delta t$ is the excitation delay time between two neighboring transducer elements in the line-array source, $z-(l-1) \Delta z$ is the separation from the $l$ th transducer element in the line-array source to the receiver, $V_{f}$ is the acoustic velocity in the borehole fluid, the derivation of $A\left(k_{z}, \omega\right)$ can be found in some previous published papers $[2,12-14]$ and $l=1,2, \ldots . . n$.

\subsection{Concept of Directivity-Weighted Coefficient and Output Signal of Receiver}

During acoustic-logging, some logging information can be obtained, such as the head-wave amplitude, and the acoustic velocities of the $P$ - and $S$-waves. The direct wave is absorbed by the sound absorption body in the logging tool. Therefore, in the following calculation of the acoustic-beam steering, we do not consider the direct wave. For the configuration of acoustic-logging as shown in Fig. (2), its acoustic-logging transmission network in time domain is shown in Fig. (3). Where, $U_{1 l}[t-(l-1) \Delta t]$ is the drivingvoltage signal for each transducer element in the line-array source, and $H[t-(l-1) \Delta t]$ is the Heaviside unit step function.

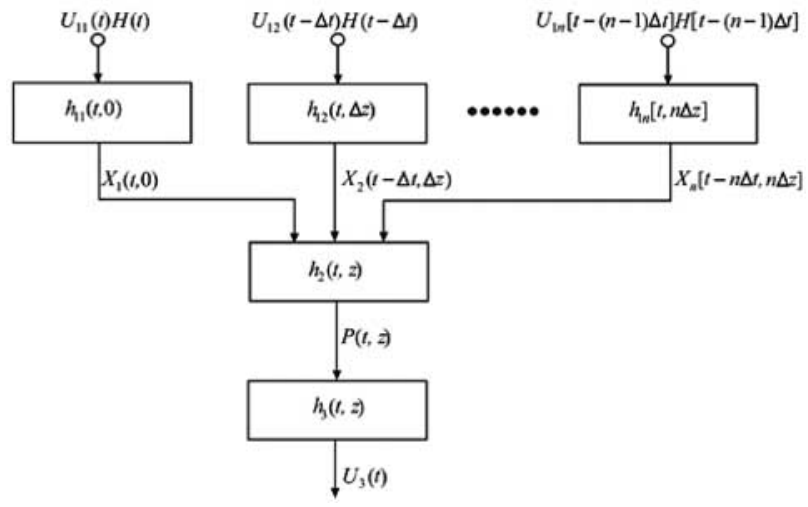

Fig. (3). Acoustic-logging transmission-network.

The output signal of the network, i.e. the electrical output signal of the receiver $J$ can be described by

$$
U_{3}(t)=\sum_{l=1}^{n} *\left\{\begin{array}{l}
U_{1 l}[t-(l-1)] H[t-(l-1) \Delta t] \\
\left.h_{1 l}[t,(l-1) \Delta z] G_{1 l}\left(\theta_{c}\right)\right\} \\
h_{2}(t, z) *\left[G_{3}\left(\theta_{c}\right) h_{3}(t, z)\right]
\end{array},\right.
$$

where, $\theta_{c}$ is the incident critical-angle from the borehole fluid to the formation around the borehole; $G_{1 l}\left(\theta_{c}\right)$ is the value of the directivity coefficient of each transducer element in the line-array source in the direction of $\theta_{c}$, and $G_{3}\left(\theta_{c}\right)$ is the value of the directivity coefficient of the receiver in the reverse direction of $\theta_{c}$. Due to the same geometrical and physical parameters for the transducer elements in the line-array source and the receiver as well as the reciprocity of the radiating and receiving directivities of the cylindrical-shell transducer, we have $G_{11}\left(\theta_{c}\right)=G_{12}\left(\theta_{c}\right)=$ $\ldots \ldots G_{1 n}\left(\theta_{c}\right)=G_{3}\left(\theta_{c}\right)=G\left(\theta_{c}\right)$. Therefore, Eq. (7) can be rewritten as 


$$
\begin{aligned}
& U_{3}(t)=\sum_{l=1}^{n} U_{1 l}[t-(l-1)] H[t-(l-1) \Delta t] \\
& h_{1 l}[t,(l-1) \Delta z] * h_{2}(t, z) * h_{3}(t, z) G^{2}\left(\theta_{c}\right)
\end{aligned}
$$

We define $G^{2}\left(\theta_{c}\right)$ as directivity-weighted coefficient, which describes the effect of the radiating and receiving directivity of the slim-hole logging tool with a line-array source and a receiver on the acoustic-logging signals.

\section{CALCULATION OF EFFECT OF DIRECTIVITY- WEIGHTED COEFFICIENT ON ACOUSTIC- LOGGING SIGNALS}

For the limitation of the geometrical-size of slim-hole, the maximal diameter of cylindrical transducer is usually no more than $30 \mathrm{~mm}$. Suppose that the source and receiver are composed of piezoelectric material PZT-5H and have the same geometrical size. Therefore we have $s_{111}^{E}=s_{311}^{E}=s_{11}^{E}$, $\varepsilon_{111}^{T}=\varepsilon_{311}^{T}=\varepsilon_{11}^{T}, \quad d_{131}=d_{331}=d_{31}, \quad \rho_{1}=\rho_{3}=\rho, \quad l_{1 t}=l_{3 t}=l_{t}$, $r_{1 b}=r_{3 b}=r_{b}$ and $H_{1}=H_{3}=H$. During the following calculations, let the wall thickness the transducer is equal to one-tenth of its outer-diameter. The compliant, dielectric and piezoelectric constants of $P Z T-5 H$ and the acoustic impedance around the transducers are shown in Table 1 [15].

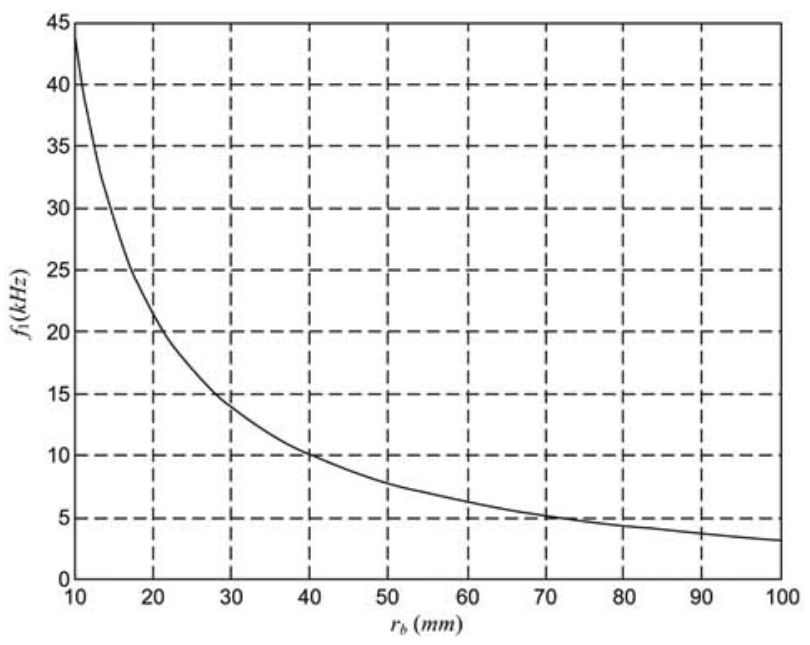

Fig. (4). Relation of load center frequency $f_{1}$ versus average radius $r_{b}$.

about $48 \mathrm{~mm}$ and $52 \mathrm{~mm}$, respectively, and the acoustic radiation area is $7841.4 \mathrm{~mm}^{2}, 3.3280$ times the former. In order to increase the radiated acoustic energy and the received acoustic signal amplitude, a line-array source of

Table 1. Physical Parameters of Piezoelectric Material PZT-5H and Acoustic Impedance of Coupling Fluid Around Transducer

\begin{tabular}{|c|c|c|c|c|}
\hline$s_{11}^{E}\left(\boldsymbol{m}^{2} / \mathbf{N}\right)$ & $\varepsilon_{11}^{T}\left(F / \mathbf{m}^{2}\right)$ & $d_{31}(\boldsymbol{m} / \boldsymbol{V})$ & $\rho\left(\mathrm{kg} / \mathrm{m}^{3}\right)$ & $Z_{m}\left(\mathrm{~kg} / \mathrm{m}^{2} \mathbf{s}\right)$ \\
\hline \hline $16.5 \times 10^{-12}$ & $3.009 \times 10^{-8}$ & $-274 \times 10^{-12}$ & 7500 & $1.221 \times 10^{6}$ \\
\hline
\end{tabular}

Eqs. (3) and (5) show that loading center frequencies of both the source and the receiver are related not only to the parameters $s_{11}^{E}, \varepsilon_{11}^{E}, d_{31}$ and $\rho$ of the piezoelectric material, and the acoustic impedance $Z_{m}$ of the coupling fluid, but also to the average radius, $r_{b}$, of the transducer (see the definitions of coefficients $x_{1}, y_{1}, x_{3}$, and $y_{3}$ in Appendixes A and B).

Inserting the parameters in Table 1 into Eq. (3) yields the relation of $f_{1}$ versus $r_{b}$ as shown in Fig. (4). Similarly, the relation between $f_{3}$ and $r_{b}$ can be obtained, and its calculation is not repeated.

From Fig. (4), it can be known that when $r_{b}$ is equal to $15 \mathrm{~mm}, f_{1}$ is equal to $29.019 \mathrm{kHz}$, and this radius is used to calculate the directivity of the transducer used in the slimhole acoustic-logging tool. In terms of Eq. (1), the calculated directivities of the cylindrical-shell transducer with different height are shown in Fig. (5).

From Fig. (5), it can be seen that for the radius of $15 \mathrm{~mm}$, the greater the $H$, the more concentrated the radiated acoustic energy in the direction perpendicular to the wall of the transducer. For the transducer used in the slim-hole logging tool, if its height $H$ is $25 \mathrm{~mm}$, the acoustic radiation area is $2356.2 \mathrm{~mm}^{2}$. The height and outer diameter of the transducer used in conventional acoustic-logging tools are adopting phase control technology can be employed. This kind of measurement system can (1) increase the effective radiation area of the acoustic source, (2) improve the radiating and receiving directivity of the slim-hole logging tool, and (3) make the radiated acoustic energy more concentrated in the direction of the incident critical-angle from the borehole fluid to the formation around the borehole.

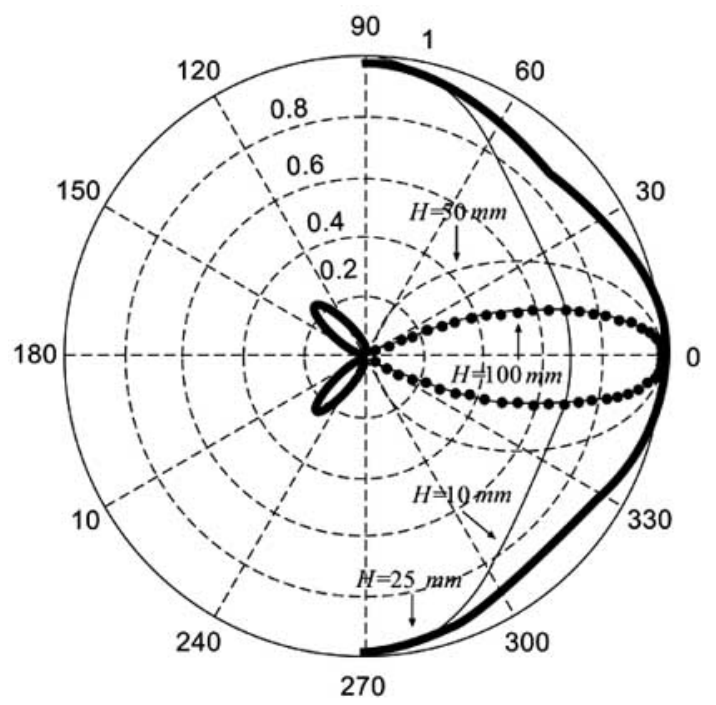

Fig. (5). Directivity of cylindrical-shell transducer with different heights. Where $r_{b}=15 \mathrm{~mm}$ and $H$ is the height of the transducer. 
If we want both the $P$-wave components of acoustic signals (which are created by all elements in the line-array source) propagating in the formation around the borehole to stack with the same phase and the $P$-wave amplitude of received acoustic signal to be the greatest, the excitation delay time between two neighboring transducer elements in the line-array source should abide by formula given by Fa and $\mathrm{Ma}$ [16]

$\Delta t_{0}=\Delta z / v_{2}$,

where, $v_{2}$ is $P$-wave velocity of the formation around the borehole.

Now, we calculate the effect of the transducer directivity on both the received acoustic-logging signal and the acoustic-beam steering efficiency of the logging tool. The chosen driving-voltage signal is a gated sinusoid given by

$U_{1}(t)=\left[H(t)-H\left(t-t_{1}\right)\right] \sin \omega_{0} t$,

where, $t_{1}$ is the window width of the sinusoid and $\omega_{0}=2 \pi f_{0}$ is the angular frequency ( $f_{0}$ is the ordinary frequency). We choose $f_{0}=f_{1}=29.019 \mathrm{kHz}, t_{1}=6 \pi / \omega_{1}$, $R=0.135 \mathrm{~m}, L=1.4 \mathrm{~m}, \Delta z=40 \mathrm{~mm}$, and the physical parameters of both the borehole fluid and the formations around the borehole as shown in Table 2.

Table 2. Physical Parameters of Borehole Fluid and Formations Around Borehole. Where $v_{p}$ is $P$-Wave Velocity and $v_{s}$ is $S$-Wave Velocity

\begin{tabular}{|c|c|c|c|}
\hline Medium & $\boldsymbol{\rho ( \boldsymbol { k g } / \boldsymbol { m } ^ { 3 } )}$ & $\boldsymbol{v}_{\boldsymbol{p}}(\boldsymbol{m} / \boldsymbol{s})$ & $\boldsymbol{v}_{\boldsymbol{s}}(\boldsymbol{m} / \boldsymbol{s})$ \\
\hline \hline Borehole fluid & 880 & 1440 & \\
\hline Formation 1 & 2100 & 2770 & 1630 \\
\hline Formation 2 & 2160 & 3500 & 1900 \\
\hline Formation 3 & 2400 & 5640 & 3200 \\
\hline
\end{tabular}

\subsection{Relation Between Received Head-Wave Amplitude and Transducer Height $H$}

For a given radius, the outer side-face of the cylindricalshell transducer polarized in its radius direction increases with its height $H$ and this outer side-face is the total radiation area of the transducer. For the calculations of the three formations shown in Table 2, let the radius of the transducer be $15 \mathrm{~mm}$ and the variation range of its height be from 10 $\mathrm{mm}$ to $75 \mathrm{~mm}$, respectively. The maximum value of the calculated head-wave amplitude is used to perform the normalization of the calculated head-wave amplitude in the variation range of the transducer height. The obtained results are called the normalized head-wave amplitude of the total radiation area. The maximum value of the ratio of the calculated head-wave amplitude to the total radiation area of the transducer is used to perform the normalization of the ratio of the calculated head-wave amplitude to the total radiation area in the variation range of the transducer height. The obtained results are called the normalized head-wave amplitude of the unit radiation area. Let the driving-voltage signal only excite the first transducer element $T_{1}$ in the linearray source, as shown in Fig. (2). The calculated relations between the head-wave amplitude of received acousticlogging signal and the transducer height $H$ are shown in Fig. (6). The curves (1) and (4) are the calculated results for formation 1, the curves (2) and (5) are those for formation 2, and the curves (3) and (6) are those for formation 3. The curves (1), (2) and (3) are the relationships between the normalized head-wave amplitude of the unit radiation area and the transducer height, and the curves (4), (5) and (6) are those between the normalized head-wave amplitude of the total radiation area and the transducer height.

For the case of the unit radiation area, the normalized head-wave amplitude decreases monotonically with increasing the transducer height $H$. For the case of the total radiation area, the maximum value of the normalized headwave amplitude occurs at an optimal transducer height. Such as, for formation 1 , at $H=0.0350 \mathrm{~m}$, the normalized headwave amplitude reaches its maximum, which is equal to 0.5105 ; for formation 2 , at $H=0.0440 \mathrm{~m}$, the normalized head-wave amplitude has its maximum, which is equal to 0.6281 ; and for formation 3 , at $H=0.0706 \mathrm{~m}$, the normalized head-wave amplitude is equal to 1 , which is the maximal value of the normalized head-wave amplitude. The calculated results show: (1) the greater the acoustic velocity of the formation, the greater the obtained head-wave amplitude value, and (2) for a given radius, a greater transducer height (or radiation area) cannot guarantee the received head-wave amplitude to be greater. The reasons are: (1) the acoustic energy radiated by the transducer with greater height is more concentrated in the direction perpendicular to the wall of the transducer, and (2) the formation with a greater acoustic velocity has a smaller incident critical-angle.

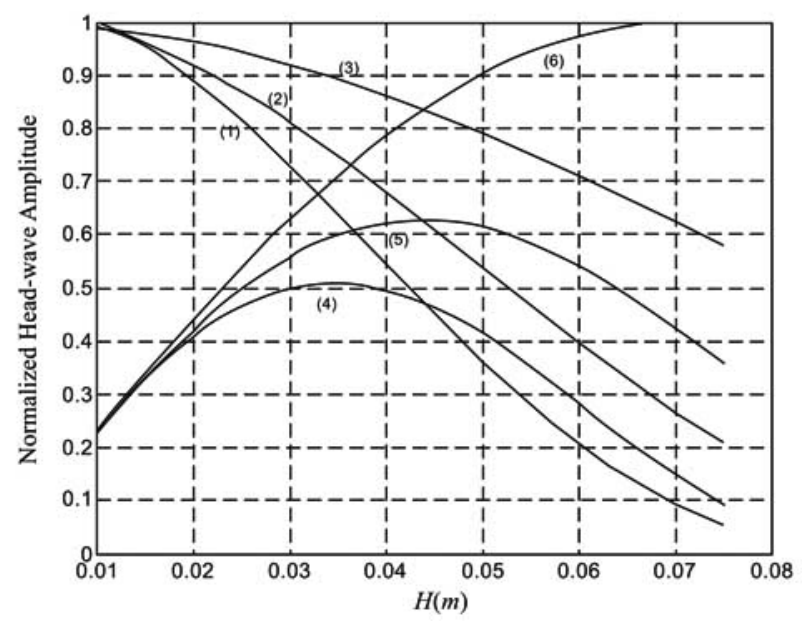

Fig. (6). Relations between normalized head-wave amplitude and transducer height $H$.

\subsection{Relations Between Received Head-Wave Amplitudes and Excitation Delay}

In this sub-section, we calculate the relation between the received head-wave amplitude and the excitation delay for 
the cases of considering and neglecting the transducer directivity. Let the line-array source consist of four transducer elements.

For Eq. (8), we use the excitation delay $\Delta t$ as variable and keep other parameters constant. For each formation shown in Table 2, we use the maximum value of the calculated head-wave amplitude for the case of neglecting the transducer directivity to perform the normalization. For the slim-hole logging tool with a line-array source and a receiver, the calculated relation between the normalized head-wave amplitude and the excitation delay are given as shown in Fig. (7).

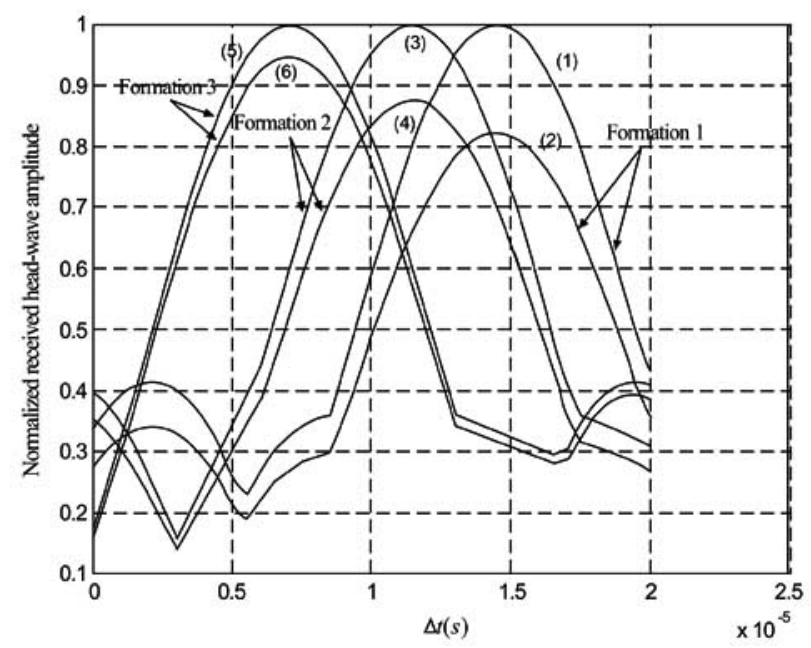

Fig. (7). Relation of head-wave amplitude versus excitation delay $\Delta t$.

The curves (1), (3) and (5) are the cases of neglecting transducer directivity, i.e. during the calculations by using Eq. (8), let $G^{2}\left(\theta_{c}\right)=1$. The curves (2), (4) and (6) are the cases of considering the transducer directivity. It is obvious that the received head-wave amplitudes for the case of considering the directivity are smaller than those for the case of neglecting the directivity. The directivity has greater effect on logging signals for the slow velocity formation than that for the fast velocity formation. The reason is that the slower velocity formation has a greater incident criticalangle. At $\Delta t=\Delta t_{0}=\Delta z / v_{p}, P$-waves created by all transducer elements in the line-array source stack with the same phase and the received head-wave amplitude is the greatest.

The calculated results shown in Fig. (7) can be converted to the radiating and receiving directivity of the slim-hole logging tool as shown in Fig. (8).

The curves (1), (2) and (3) in Fig. (8) are the cases for formations 1, 2 and 3, respectively. It can be seen from Fig. (8) that the higher the acoustic velocity of the formation, the smaller the acoustic-beam steering angle. Due to the smaller incident critical-angle for the fast velocity formation around the borehole, the effect of the transducer directivity on the received head-wave amplitude for the fast velocity formation is smaller than that for the slow velocity formation.



Fig. (8). Radiating and receiving directivity of the logging tool with a line-array source and a receiver.

\subsection{Relation of Steered Acoustic-Beam Width Versus Interval Between Two Neighboring Transducer Elements}

We use the formation 2 as example to calculate the relation of the steered acoustic-beam width versus the interval $\Delta z$ between two neighboring transducer elements in the line-array source. When $\Delta z$ is equal to $25 \mathrm{~mm}, 35 \mathrm{~mm}$ and $45 \mathrm{~mm}$, respectively, the calculated radiating and receiving directivities of the slim-hole logging tool are shown in Fig. (9).

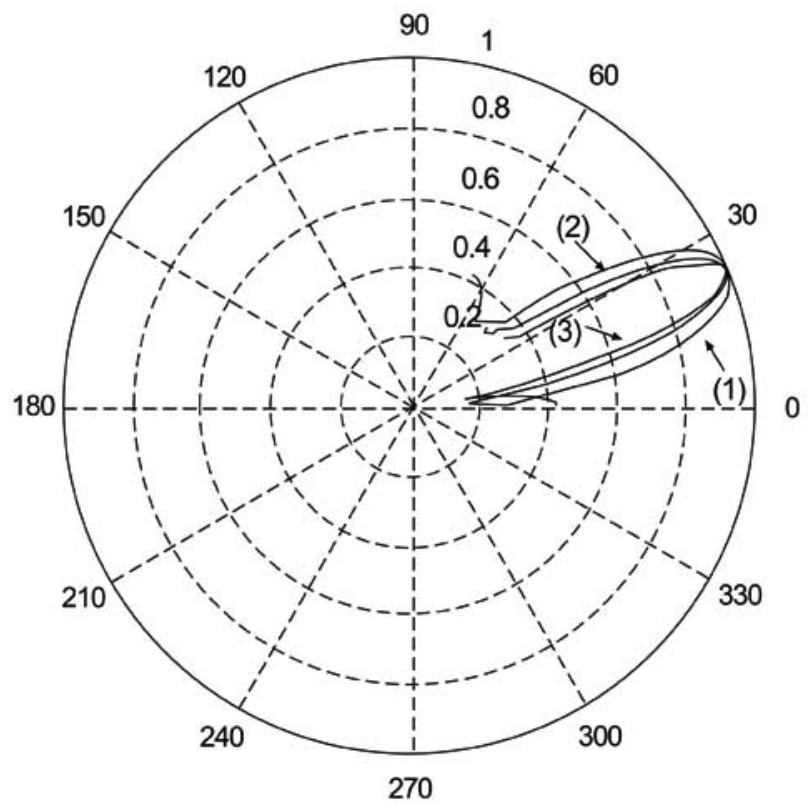

Fig. (9). Relation of steered acoustic-beam width versus $\Delta z$.

In Fig. (9), curve (1) is the radiating and receiving directivity for the case of $\Delta z=25 \mathrm{~mm}$, curve (2) is for the case of $\Delta z=35 \mathrm{~mm}$, and curve (3) is for the case of $\Delta z=45$ $\mathrm{mm}$. The above calculated results show that the greater $\Delta z$, the narrower the steered acoustic-beam width and the more 
concentrated the radiated $P$-wave energy in the direction of $\theta_{c}$.

\subsection{Relation of Head-Wave Amplitude Versus Transducer Element Number in Line-Array Source}

For the slim-hole logging tool with a line-array source and a receiver, when the $P$-wave velocity of the formation 3 is used to adjust the excitation delay of the line-array source, the calculated relation between the transducer element number in the line-array source and the normalized received head-wave amplitude is shown in Table 3. Where $H$ is equal to $25 \mathrm{~mm}, n$ is the transducer element number, HWA1 and HWA2 are the received head-wave amplitudes for the cases of neglecting and considering transducer directivity, respectively.

Table 3. Relations of Transducer Element Number Versus Received Head-Wave Amplitude

\begin{tabular}{|c|c|c|c|c|}
\hline $\boldsymbol{n}$ & $\mathbf{1}$ & $\mathbf{2}$ & $\mathbf{3}$ & $\mathbf{4}$ \\
\hline \hline HWA1 & 0.2445 & 0.4925 & 0.7443 & 1.0000 \\
\hline HWA2 & 0.2318 & 0.4670 & 0.7057 & 0.9482 \\
\hline
\end{tabular}

From Table 3, it can be seen that if the transducer element number in the line-array source is $n$, the received head-wave amplitude is approximately equal to $n$ times the received head-wave amplitude for the case of a single transducer element emission. The directivity-weighted coefficient makes the received head-wave amplitude decrease to a certain extent.

\section{CONCLUSIONS}

From the above calculations and analysis, some concluding remarks can be reached.

1. We put forward the concept of the radiating and receiving directivity-weighted coefficient for the slim-hole logging tool with a line-array source and a receiver.

2. On the basis of acoustic-logging transmission network model, we calculate and analyze the effects of the geometrical-size of cylindrical-shell transducer on the head-wave amplitude of received acousticlogging signal.

3. The directivity-weighted coefficient has greater effect on acoustic-logging signal for slow velocity formation than that for fast velocity formation.

4. Using the $P$-wave velocity of the formation around the borehole to adjust the excitation delay of the linearray source can make the received head-wave amplitude increase greatly.

5. When the radius of the transducer element is $15 \mathrm{~mm}$, and its height is equal to $35 \mathrm{~mm}, 44 \mathrm{~mm}$ and $70.6 \mathrm{~mm}$, respectively, the received head-wave amplitudes can reach their maximal value for formations 1,2 and 3 shown in Table 2.
6. The greater the interval $\Delta z$ between two neighboring transducer elements in the line-array source, the narrower the steered acoustic-beam.

7. The calculated results and analysis method may be useful for improving the radiating and receiving directivity of the slim-hole logging tool.

\section{ACKNOWLEDGEMENTS}

This work is supported by National Natural Science Foundation, China (Grant No. 40974078), US Department of Energy (Grant No. DE-FC26-08NT0005643), the Natural Science Foundation of Shaanxi Province, China (Grant No. 2007D15), and North Dakota Industrial Commission with five industrial companies: Encore Acquisition, Hess, Marathon Oil, St. Mary Land \& Exploration, and Whiting Petroleum (Grant No. NDIC-G015-031).

\section{APPENDIX A}

When the cylindrical-shell transducer is used as a source, its electrical-acoustic transmission network is shown in Fig. (1a), and Fig. (A) is its $s$-domain equivalent.

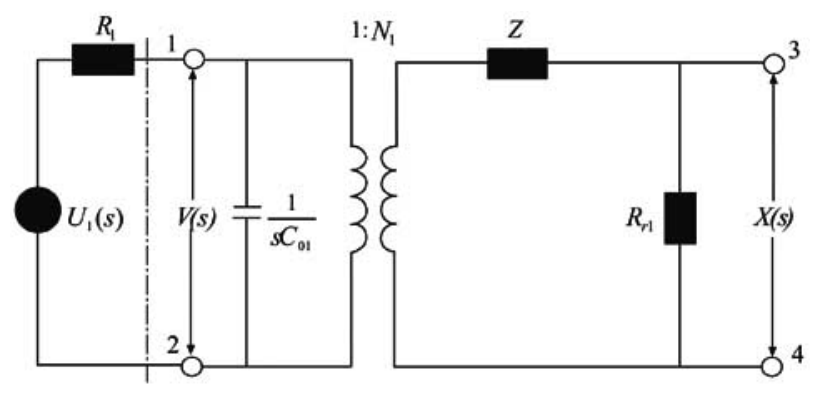

Fig. (A) Electrical-acoustic transmission network of cylindricalshell transducer in s-domain. Where, $Z=s\left(m_{1}+m_{r 1}\right)+R_{m 1}+1 / s C_{m 1}$.

Now, we derive both the electrical-acoustic impulse response and the loading center frequency for the source. From Fig. (A), we can get

$H_{1}(s)=\frac{d_{1} s}{s^{3}+a_{1} s^{2}+b_{1} s+c_{1}}$,

where, $a_{1}=b_{11} / a_{11}, b_{1}=c_{11} / a_{11}, c_{1}=d_{11} / a_{11}, d_{1}=e_{11} / a_{11}$,

$a_{11}=R_{1} C_{01} C_{m 1} \tilde{M}_{1}, b_{11}=R_{1} C_{01} C_{m 1} \tilde{R}_{1}+C_{m 1} \tilde{M}_{1}$,

$c_{11}=N^{2} R_{1} C_{m 1}+R_{1} C_{01}+\tilde{R}_{1} C_{m 1}, \quad d_{11}=1$,

$e_{11}=N_{1} R_{r 1} C_{m 1}, \quad \tilde{M}_{1}=m_{1}+m_{r 1}$ and $\tilde{R}_{1}=R_{m 1}+R_{r 1}$.

By inserting the physical and geometrical parameters of the transducer and the acoustic impedance of the coupling medium (transformer oil) into Eq. (A-1), the calculated three extreme points $s_{1}, s_{2}$ and $s_{3}$ are all located in the left part of the complex plane. Therefore, $H_{1}(s)=\left.H_{1}(j \omega)\right|_{s=j \omega}$ and the electrical-acoustic transmission function of the cylindrical-shell transducer can be written as

$H_{1}(\omega)=\frac{j \omega d_{1}}{-j \omega^{3}-\omega^{2} a_{1}+j \omega b_{1}+c_{1}}$. 
The three extreme points of Eq. (A-1) are

$s_{1}=-\alpha_{1}$

$s_{2}=-\beta_{1}+j \omega_{1}$,

$s_{3}=-\beta_{1}-j \omega_{1}$,

where, $A_{1}=\left(x_{1}+y_{1}\right) / 2, B_{1}=\left(x_{1}-y_{1}\right) / 2$,

$x_{1}=\sqrt[3]{-q_{1} / 2+\sqrt{D_{1}}}, y_{1}=\sqrt[3]{-q_{1} / 2-\sqrt{D_{1}}}$,

$D_{1}=\left(p_{1} / 3\right)^{3}+\left(q_{1} / 2\right)^{2}, p_{1}=b_{1}-a_{1}^{2} / 3$,

$q_{1}=c_{1}+2 a_{1}^{3} / 27-a_{1} b_{1} / 3, \alpha_{1}=a_{1} / 3-2 A_{1}, \beta_{1}=a_{1} / 3+A_{1}$,

and $\omega_{1}=\sqrt{3} B_{1}$.

Therefore, Eq. (A-1) can be rewritten as

$H_{1}(s)=\frac{e_{1} s}{\left(s-s_{1}\right)\left(s-s_{2}\right)\left(s-s_{3}\right)}=H_{11}(s)+H_{12}(s)+$

the conjugate term of $H_{12}(s)$

$=\frac{K_{1}}{s+\alpha_{1}}+\frac{K_{2}}{s+\beta_{1}-j \omega_{1}}+$ the conjugate term of $H_{12}(s)$.

By performing Laplace transform on $H_{11}(s), H_{12}(s)$ and the conjugate term of $H_{12}(s)$, we can get the electricalacoustic impulse response of the transducer as follows

$h_{1}(t)=h_{11}(t)+h_{12}(t)=K_{11} e^{-\alpha_{1} t}+K_{12} e^{-\beta_{1} t} \cos \left(\omega_{1} t+\theta_{1}\right)$,

where,

$K_{11}=-d_{1} \alpha_{1} /\left[\left(-\alpha_{1}+\beta_{1}\right)^{2}+\omega_{1}^{2}\right], K_{12}=d_{1} E_{1} /\left(\alpha_{1}^{2}-\beta_{1}^{2}+\omega_{1}^{2}\right)$,

$\theta_{1}=\operatorname{arctg}\left[\left(\beta_{1}^{2}-\omega_{1}^{2}\right) /\left(\omega_{1} \alpha_{1}\right)\right]$, and

$E_{1}=\sqrt{\alpha_{1}^{2}+\left[\left(\beta_{1}^{2}-\omega_{1}^{2}\right) / \omega_{1}\right]^{2}}$.

From Eq. (A-7) and the above coefficients, the loading center frequencies of the source can be written as

$f_{1}=\omega_{1} / 2 \pi=\sqrt{3} B_{1} / 2 \pi=\sqrt{3}\left(x_{1}-y_{1}\right) / 4 \pi$.

\section{APPENDIX B}

When the cylindrical-shell transducer is used as a receiver, Fig. (1b) is the acoustic-electrical transmission network of the receiver, and its $s$-domain equivalent is shown in Fig. (B).

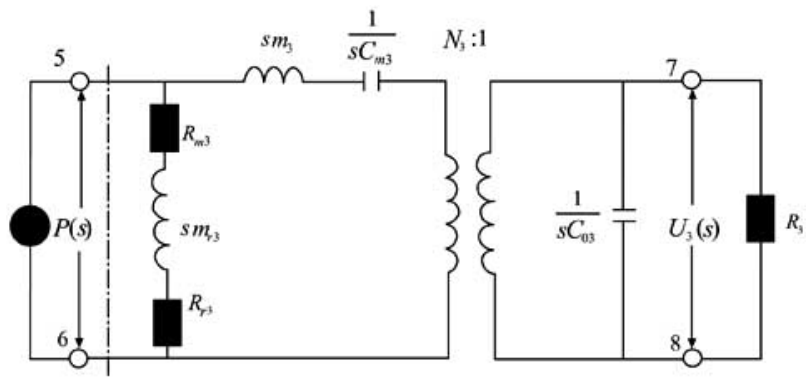

Fig. (B). Acoustic-electrical transmission network of cylindricalshell transducer in s-domain.
In the following, we derive both the acoustic-electrical impulse response and the loading center frequency for the receiver. From Fig. (B), we can get

$H_{3}(s)=\frac{d_{3} s}{s^{3}+a_{3} s^{2}+b_{3} s+c_{3}}$

where, $a_{3}=b_{33} / a_{33}, b_{3}=c_{33} / a_{33}, c_{3}=d_{33} / a_{33}$ and

$d_{3}=e_{33} / a_{33}, a_{33}=C_{03} m_{3} C_{m 3} R_{3}, b_{33}=m_{3} C_{m 3}$,

$c_{33}=R_{3}\left(C_{03}+N_{3}^{2} C_{m 3}\right), d_{33}=1$, and $e_{33}=N_{3} R_{3} C_{m 3}$,

By repeating the derivation method described above, we can get the acoustic-electrical transmission function and impulse response of the transducer as follows

$H_{3}(\omega)=\frac{j \omega d_{3}}{-j \omega^{3}-\omega^{2} a_{3}+j \omega b_{3}+c_{3}}$,

and

$h_{3}(t)=h_{31}(t)+h_{32}(t)=K_{31} e^{-\alpha_{3} t}+K_{32} e^{-\beta_{3} t} \cos \left(\omega_{3} t+\theta_{3}\right)$,

where, $A_{3}=\left(x_{3}+y_{3}\right) / 2, B_{3}=\left(x_{3}-y_{3}\right) / 2$,

$\alpha_{3}=a_{3} / 3-2 A_{3}, \beta_{3}=a_{3} / 3+A_{3}, \omega_{3}=\sqrt{3} B_{3}$,

$x_{3}=\sqrt[3]{-q_{3} / 2+\sqrt{D_{3}}}, y_{3}=\sqrt[3]{-q_{3} / 2-\sqrt{D_{3}}}$

$D_{3}=\left(p_{3} / 3\right)^{3}+\left(q_{3} / 2\right)^{2}, p_{3}=b_{3}-a_{3}^{2} / 3$,

$q_{3}=c_{3}+2 a_{3}^{3} / 27-a_{3} b_{3} / 3, K_{31}=-d_{3} \alpha_{3} /\left[\left(-\alpha_{3}+\beta_{3}\right)^{2}+\omega_{3}^{2}\right]$

, $K_{32}=d_{3} E_{3} /\left(\alpha_{3}^{2}-\beta_{3}^{2}+\omega_{3}^{2}\right), \theta_{3}=\operatorname{arctg}\left[\left(\beta_{3}^{2}-\omega_{3}^{2}\right) /\left(\omega_{3} \alpha_{3}\right)\right]$, and $E_{3}=\sqrt{\alpha_{3}^{2}+\left[\left(\beta_{3}^{2}-\omega_{3}^{2}\right) / \omega_{3}\right]^{2}}$.

From Eq. (B-3) and the above coefficients, the loading center frequencies of the receiver can be expressed by

$f_{3}=\omega_{3} / 2 \pi=\sqrt{3} B_{3} / 2 \pi=\sqrt{3}\left(x_{3}-y_{3}\right) / 4 \pi$.

\section{REFERENCES}

[1] Aki K, Rechards PG. Quantitative Seismology, Theory and Methods. San Francisco: W. H. Freeman 1980.

[2] Tsang L, Rader D. Numerical evaluation of the transient acoustic waveform due to a point source in a fluid-filled bore hole. Geophysics 1979; 44: 1706-20.

[3] Renlie L, Raaen AM. Acoustic wave propagation in fluid-filled borehole surrounded by a formation with stress-relief induced anisotropy. Geophysics 1993; 58: 1257-69.

[4] Gibson Jr RL, Peng C. Low- and high-frequency radiation from seismic sources in cased boreholes. Geophysics 1994; 59: 1780-85.

[5] Fa L, Castagna JP, Hovem JM. Derivation and simulation of source function for acoustic-logging. IEEE Ultrasonics Symposium Proceedings; November 1999; Lake Tahoe, USA 1999.

[6] Fa L, Castagna JP, Hovem JM, Dong D. An acoustic-logging transmission-network model. J Acoust Soc Am 2002; 111: 2158 65 .

[7] Fa L, Castagna JP, Suarez-Rivera R, Sun P. An acoustic-logging transmission-network model (continued): addition and multiplication ALTNs. J Acoust Soc Am 2003; 113: 2698-703.

[8] He Z, Zhao Y. Fundamentals of Acoustic Theory. Bejing: China National Industrial Press 1980.

[9] Fa L, Wang H, Ma M. Acoustic Array Transmitting, Receiving Sondes and Processing Method of Received Full Waves. Transactions of International Conference on Earth Physics Logging; May 1990; Beijing, China 1990.

[10] Fa L, Lin F, Chen W. Source function derivation and property analysis of ceramic ring transducer for petroleum exploration. Acta Geophys 1996; 39(Suppl): 387-99. 
[11] Kurkjian AL. Numerical computation of individual far-field arrivals excited by an acoustic source in a borehole. Geophysics 1985; 50: 852-66.

[12] Biot MA. Propagation of elastic waves in a cylindrical bore containing a fluid. J Appl Phys 1952; 23: 997-1005.

[13] Roever W, Rosenbaum J, Vining, T. Acoustic waves from an impulsive source in a fluid-filled borehole. J Acoust Soc Am 1974; 55: 1144-57.
[14] Peterson EW. Acoustic wave propagation along a fluid-filled cylinder. J Acoust Soc Am 1974; 45: 3334-50.

[15] Zhang P, Zhang Z. Measurement for piezoelectric material. Beijing, China: National Defense Press 1983.

[16] Fa L, Ma H. Design of a new type of array transmitting sonic sonde. Acta Petrol Sin 1991; 3: 52-57.

Received: May 5, 2009

Revised: October 30, 2009

Accepted: March 1, 2010

(C) Fa et al.; Licensee Bentham Open.

This is an open access article licensed under the terms of the Creative Commons Attribution Non-Commercial License (http://creativecommons.org/licenses/by$\mathrm{nc} / 3.0 /$ ) which permits unrestricted, non-commercial use, distribution and reproduction in any medium, provided the work is properly cited. 